INPLASY

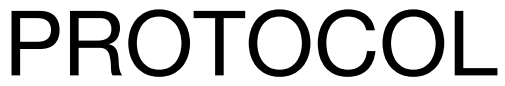

To cite: Xue et al. Acupuncture and related therapies for stable angina pectoris: a protocol for network meta-analysis. Inplasy protocol 2020110035. doi:

10.37766/inplasy2020.11.0035

Received: 09 November 2020

Published: 09 November 2020

Corresponding author:

Qiqi Yang

821920266@qq.com

Author Affiliation:

Hubei University of Chinese Medicine, No. 01 Tanhualin, Wuchang District, Wuhan, Hubei 430061, China

Support: Not available.

Review Stage at time of this submission: Preliminary searches.

Conflicts of interest:

None.

\section{Acupuncture and related therapies for stable angina pectoris: a protocol for network meta-analysis}

\author{
Xue, YX1; Yang, QQ2; Zhang, XL3; Zhang, YJ4; Huang, W5.
}

Review question / Objective: The objective of this systematic review is to evaluate the comparative effectiveness of different acupuncture and related therapies in the treatment of patients with stable angina pectoris.

Condition being studied: Stable angina pectoris is a form of chronic heart disease associated with ill health and increased death rates, which greatly endangers patient's life quality and longevity. Acupuncture, as a key component of traditional Chinese medicine (TCM), has been one of the most widely used complementary therapies in many countries. Previous systematic reviews have shown that acupuncture may safely and effectively improve some symptoms such as attack frequency in patients with stable angina pectoris. However, due to the wide variety of acupuncture-related therapies and the lack of direct comparison between different acupuncture related therapies, it is not conducive to clinical promotion and the choice of the best treatment method. Hence, we will conduct this network meta-analysis to analyse both direct and indirect comparisons of different acupuncture and related therapies for treating stable angina pectoris.

INPLASY registration number: This protocol was registered with the International Platform of Registered Systematic Review and Meta-Analysis Protocols (INPLASY) on 09 November 2020 and was last updated on 09 November 2020 (registration number INPLASY2020110035).

\section{INTRODUCTION}

Review question / Objective: The objective of this systematic review is to evaluate the comparative effectiveness of different acupuncture and related therapies in the treatment of patients with stable angina pectoris.
Condition being studied: Stable angina pectoris is a form of chronic heart disease associated with ill health and increased death rates, which greatly endangers patient's life quality and longevity. Acupuncture, as a key component of traditional Chinese medicine (TCM), has been one of the most widely used 
complementary therapies in many countries. Previous systematic reviews have shown that acupuncture may safely and effectively improve some symptoms such as attack frequency in patients with stable angina pectoris. However, due to the wide variety of acupuncture-related therapies and the lack of direct comparison between different acupuncture related therapies, it is not conducive to clinical promotion and the choice of the best treatment method. Hence, we will conduct this network meta-analysis to analyse both direct and indirect comparisons of different acupuncture and related therapies for treating stable angina pectoris.

\section{METHODS}

Search strategy: We will search the following electronic databases without restrictions for language or publication status: PubMed, EMBASE, The Cochrane Library, Web of Science, Chinese National Knowledge Infrastructure (CNKI), Wanfang database, Chinese Science and Technology Periodical Database (VIP) and China Biology Medicine Database (CBM) from inception to November 2020. The search terms were used as follows: "acupuncture OR acupuncture therapy OR electroacupuncture OR silver needle OR warm needling OR moxibustion OR acupoint injection OR acupoint application OR acupoint catgut embedding OR auricular acupuncture" AND " stable angina pectoris" AND "randomized controlled trials".Besides, the previous relevant reviews conducted on acupuncture-related therapies for stable angina pectoris and reference lists of included studies will also be searched. And the searches will be rerun just before the final analyses to retrieve the most recent studies eligible for inclusion.

Participant or population: Inclusion: Men and women who had been diagnosed with stable angina pectoris according to the diagnostic criteria of ACC/AHA angina pectoris, regardless of their age, race or gender. Exclusion: 1) People with unstable angina (pain at rest) and those with refractory angina for whom revascu- larisation was planned. 2)Participants who are not appropriate to receive acupuncture therapy, such as pregnant or lactating women. 3)People with additional severe diseases.

Intervention: Acupuncture and related therapies including but not limited to acupuncture, electric acupuncture, warm needling, moxibustion, acupoint injection, acupoint application, acupoint catgut embedding, auricular acupuncture, regardless of time of treatment and amount of stimulation. Acupuncture therapies combined with herbs or massage will be excluded.

Comparator: Control intervention will be limited to sham acupuncture or placebo, routine care, conventional drugs. When studies combine acupuncture therapies with other active therapy, both the experimental and the control groups are required to use the same active therapy.

Study designs to be included: Randomized controlled trials (RCT).

Eligibility criteria: The eligibility criteria will be achieved when all literatures meet the requirements above. In addition, study meeting the following criteria will be excluded: 1. Case report, cross-sectional studies, comments, cohort studies, animal experiments, and reviews will be excluded. 2. Incomplete data or information.3. Duplicate publication. 4. No predetermined outcome index.

Information sources: We will search the following electronic databases without restrictions for language or publication status: PubMed, EMBASE, The Cochrane Library, Web of Science, Chinese National Knowledge Infrastructure (CNKI), Wanfang database, Chinese Science and Technology Periodical Database (VIP) and China Biology Medicine Database (CBM) from inception to November 2020. Besides, the previous relevant reviews conducted on acupuncture-related therapies for stable angina pectoris and reference lists of included studies will also be searched. If 
there are ambiguous or unreported data, we will contact the corresponding authors for the missing data to get specific information by email.

Main outcome(s): Angina attack frequency (The number of weekly angina attacks ): mean difference (MD) with $95 \%$ confidence intervals (Cl).

Additional outcome(s): 1. ECG changes (mainly ST-segment depression); 2. Angina pain intensity (assessed by visual analogue scale); 3. Performance on the Six-Minute Walk Test (6-MWT); 4. Reported adverse events.

Data management: All reviewers will receive professional training to understand the objective and process of the review before the selection of studies. All the retrieved studies will be managed with EndnoteX7, and the duplicated studies will be discarded.

Quality assessment / Risk of bias analysis: The risk of bias will be performed by two raters independently (YQQ and ZXL) based on the Cochrane risk of bias tool. It includes seven specific domains: (1) random sequence generation, (2) allocation concealment, (3) blinding of participants and personnel, (4) blinding of outcome data, (5) incomplete outcome data, (6) selective reporting and (7) other bias. We will manage to reach consensus between two raters on condition that there are any disagreement when assessing the risk of bias of the studies. If necessary, a third rater $(X Y X)$ will resolve the disagreement.

Strategy of data synthesis: For each direct treatment comparison, if no less than two RCTs, we will perform pairwise metaanalyses. We will use the odds ratio with 95\% confidence intervals $(\mathrm{Cl})$ to assess the effect size of dichotomous variables, while the effect size of continuous variables will be assessed using the mean difference (MD). Heterogeneity between the studies in effect measures will be assessed using the $\mathrm{I}^{2}$ statistic, and we will consider an $\mathrm{I}^{2}$ value greater than $50 \%\left(I^{2}>50 \%\right)$ as being indicative of substantial heterogeneity, and in this case, a random-effects model will be applied to process the data. Otherwise, we choose a fixed-effects model $\left(I^{2} \leq 50 \%\right)$. When the results are substantial heterogeneity or considerable heterogeneity, sensitivity analysis and subgroup analysis will be made to explore possible sources. GeMTC 0.14.3 software will be used to analyze the data. STATA 13.0 and WinBUGS 1.4.3 will be used to perform NMA to synthesize direct and indirect evidence. The convergence of the stimulation is tested by the potential scale reduction parameter, when the closer potential scale reduction parameter will be to 1 , the better the model convergence is and more reliable the conclusion is. The selection of the final model will depend on the deviance information criterion value. Generally, a model with a smaller deviance information criterion value is better. The effect size is expressed in $95 \% \mathrm{Cl}$, and the numerical variable is expressed as SMD. The treatment level for each result will operate on the cumulative sorting curve (SUCRA) interface. The evidence relationship incorporated into the study will be calculated by STATA. If there is a "closed loop," the node splitting method will be used to evaluate the inconsistency of each loop.

Subgroup analysis: When considerable heterogeneity is detected in a previous analysis, a subgroup analysis will be performed if necessary. Subgroup analyses on the following factors will be conducted to assess heterogeneity as well as possible: age, duration of disease, type of acupuncture, the treatment of control group.

Sensibility analysis: Before selecting model, sensitivity analysis will be performed if sufficient studies are available.

Country(ies) involved: China.

Keywords: acupuncture, acupuncture and related therapies, stable angina pectoris, protocol, network meta-analysis. 
Contributions of each author:

Author 1 - Yixuan Xue - The author drafted the manuscript.

Email: 654456965@qq.com

Author 2 - Qiqi Yang - The author provided statistical expertise.

Email: 821920266@qq.com

Author 3 - Xiaolei Zhang - The author contributed to the development of the selection criteria, and the risk of bias assessment strategy.

Email: 1823129759@qq.com

Author 4 - Yanji Zhang - The author is responsible for data curation.

Email: 499149302@qq.com

Author 5 - Wei Huang - The author read, provided feedback and approved the final manuscript.

Email: 24231637@qq.com 